\title{
Rayleigh-Bénard Convection in Ferrofluids in the Microgravity Environment
}

\author{
H. A. Jasmine* \\ Department of Mathematics, University of Rajshahi, Rajshahi, Bangladesh \\ Received 20 April 2016, accepted in final revised form 27 June 2016
}

\begin{abstract}
We have investigated stability of a ferrofluid in microgravity environment. We consider the effect of the spin viscosity, vortex viscosity, and magnetization relaxation. The eigenvalue problem is solved by employing the Chebyshev pseudospectral method and the results are discussed for all three boundary conditions: free-free, rigid-free and rigidrigid. In the microgravity environment, the ferrofluid is more resilient to convection and, in general, for all boundary conditions requires higher temperature gradient for the threshold of the convection. It is found that a ferrofluid in microgravity environment is more stable, not only as compared to the case when gravity is present, but also in the pure viscous case.
\end{abstract}

Keywords: Ferrofluids; Stability; Convection; Microgravity environment; magnetic field.

(C) 2016 JSR Publications. ISSN: 2070-0237 (Print); 2070-0245 (Online). All rights reserved. doi: http://dx.doi.org/10.3329/jsr.v8i3.27438 J. Sci. Res. 8 (3), 309-319 (2016)

\section{Introduction}

A ferrofluid is a liquid that becomes strongly magnetized in the presence of a magnetic field. A liquid that consists of a colloidal suspension of ferromagnetic particles and that becomes strongly polarized in the presence of a magnetic field. Ferrofluids are colloidal liquids made of nanoscale ferromagnetic particles suspended in a carrier fluid. Each tiny particle is thoroughly coated with a surfactant to inhibit clumping. Large ferromagnetic particles can be ripped out of the homogeneous colloidal mixture, forming a separate clump of magnetic dust when exposed to strong magnetic fields. Ferrofluids do not retain the polarization once the magnetic field is removed. Several applications of ferrofluids includ their use in liquid seals in rotary shafts for vacuum systems and in hard disk drives of personal computers. Ferrofluids are also used in

* Corresponding author: hosne_jasmine@ hotmail.com 
cooling and damping of loud speakers, in shock absorbers and in jet printing of magnetic inks [1]. Several physical phenomena, which were not explained hitherto, were explained by this theory. This included the increase of the viscosity with an increase in the d.c. magnetic field [2] and the decrease of viscosity (sometimes termed as negative viscosity) with the increase of high frequency a.c. magnetic field [3]. The experiments in both these two later papers were limited to slow laminar flow. This theory, however, is based upon a single particle calculation and thus could only be applied to dilute ferrofluids.

The general development of more sophisticated ferrofluid equations, which not only employs the concepts of internal rotation, magnetization relaxation, etc. but also introduce the role of asymmetric stress tensor, have been proposed by Felderhof and Kroh [4]. In some sense all these theories are generalizations of the ideas introduced earlier by Dahler and Scriven [5] and Condiff and Dahler [6] in the studies of structured continua and polarizable molecular fluids, respectively. Shizawa and Tanahashi [7] combined the effects of electrically conducting magnetic fluids with micropolar theory. Felderhof and Kroh [4], following the framework laid out in deGroot and Mazur [8], employed irreversible thermodynamics to arrive at the general set of equations. More recently, Rosensweig [9] has developed the governing equations derived on the basis of dynamic balance relationships and thermodynamic considerations. In this paper the author also demonstrates how the equations proposed in the earlier studies by Neuringer and Rosensweig [10] are related to the equations developed by him. We point out that there is quite a bit of similarity between the final form of equations proposed by Felderhof and Kroh [4]. In the present paper we have employed the general equations suggested by Stile et al. [11].

One of the interesting problems often studied in all the fluid dynamic theories is the thermal instability problem in a fluid layer heated from below, the so called Rayleigh-Bénard convection problem. Chandrasekhar [12] has given an excellent account of the linear stability of this problem in viscous fluids. In the theory of ferrofluid by Neuringer and Rosensweig [10], the above problem has been studied extensively, as discussed below, by a number of writers. Finlayson [13] was the first author to study convective instability problem of a ferrofluid layer heated from below in the presence of a uniform magnetic field. He discussed the linear stability problem for both shear free and rigid-rigid boundaries and predicted that convection could also be driven by the magnetic forces alone, in the absence of gravity. Gotoh and Yamada [14] studied the same problem by assuming the magnetic fluid is confined between two magnetic pole pieces. Stiles and Kagan [15] discussed the convective instability in a strong magnetic field while Blennerhasset et al. [16] and Stiles et al. [11] discussed the heat transfer aspects through strongly and weakly magnetized ferrofluids respectively. Sunil and Mahajan [17] employed the energy method to study the nonlinear stability of this problem. The thermal convection problem in a rotating layer of a ferrofluid has been studied, respectively, by Gupta et al. [18], Venkatasubramanian et al. [19]. The last two references also have discussed the 
weakly nonlinear analysis. Some other aspects of the thermal convection problem in the above model have also been considered by Pérez et al. [20]. Finally, we point out that some interesting aspects of the convection problem in an oscillatory magnetic field have also been studied by Kaloni and Lou [21] and Matura and Lücke [22]. There have been some experimental studies carried out by Schwab et al. [23] related to thermal convection in ferrofluids.

This section two important remarks are in order. The first is concerned about considering ferrofluids as a binary mixture. Two representative studies in this direction are those of Shliomis [24]. Both these studies have been carried out using the constant viscosity model [10]. Both studies relate the pronounced Sorret effect and the magnetophoresis effects to be the cause of considering ferrofluid as a binary mixture. Shliomis [24] however, stated that because of considerable smallness of concentration diffusion coefficient, as compared to the thermal diffusion coefficient, the influence of concentration gradient is possible only when the temperature difference is allowed to increase very slowly so that mass diffusion develops and remains undisturbed. If the temperature difference increases much faster than the limit imposed by concentration diffusion, the ferrofluid will behave as a single component fluid. Ghofrani et al. [25] investigated experimentally forced convection heat transfer of an aqueous ferrofluid flow passing through a circular copper tube in the presence of an alternating magnetic field. The effects of magnetic field, volume concentration, and Reynolds number on the convective heat transfer coefficient were widely examined leading to the determination of optimum conditions. Increase in the alternating magnetic field frequency and the volume fraction led to better heat transfer enhancement. The effect of the magnetic field in low Reynolds numbers was higher, and a maximum of $27.6 \%$ enhancement in the convection heat transfer was observed. Radha et al. [26] reported the experimental observation of diffraction patterns in a ferrofluid under the effect of magnetic field and gravity. The diffraction pattern showed a variation at different depths of the sample in the absence of the magnetic field and when the magnetic field is applied. The patterns also exhibited a change in the shape and size with variation of the external field. This effect arises due to thermally induced self-diffraction under the influence of gravity and external magnetic field. Recently Jasmine [27] investigated the linear thermoconvective stability of ferrofluid in presence of uniform magnetic field with gravity for rigid-rigid boundary condition case only.

In this paper we have used boundary conditions: free-free, rigid-free and rigidrigid, and the fluid selected is Ester I base ferrofluid. The solution, in each case, has been obtained numerically. In general it is noted that the pattern of values remains similar to be obtained in the case of a viscous fluid. The critical values are the lowest for the free-free boundary condition and the highest for the rigid-rigid case. Moreover, the critical values of $R a_{c}$ and $R a_{m, c}$ are field-dependent. It is of some interest to determine the stability character in an environment when gravity effects can be neglected, i.e. when effects of magnetic field alone are present (e.g. international apace station). In the case when magnetic mechanism alone is considered to induce 
convection, the values of $R a_{m f, c}$, the critical magnetic Rayleigh number (in the microgravity environment), for three boundary conditions, in the case of Ester base I ferrofluid are discussed.

\section{Governing Equations}

We consider the three dimensional flow of an incompressible ferrofluid. The continuity and momentum equations are

$$
\begin{aligned}
& \nabla \cdot \boldsymbol{u}=\mathbf{0} \\
& \rho \frac{\boldsymbol{D u}}{D t}=-\nabla \mathrm{P}+(\mu+\xi) \nabla^{2} \mathbf{u}+\boldsymbol{\mu}_{\mathbf{0}}(\mathbf{M} \cdot \nabla) \mathbf{H}+2 \xi(\nabla \times \mathbf{\Omega})-\rho \mathrm{g} \hat{\mathbf{k}}
\end{aligned}
$$

where $u=(u, v, w)$ is the velocity field, with radial, tangential and vertical components $u, v$ and $w$, respectively. $\frac{D}{D t}=\frac{\partial}{\partial t}+\boldsymbol{u} . \nabla, \rho$ is the density. $\mathrm{P}=\mathrm{p}+\frac{1}{2} \mu_{0} \mathrm{H}^{2}$ is the generalized pressure, $t$ is time, $g$ the gravitational body force, $\mu$ is the viscosity, $\xi$ is the vortex viscosity, $H$ is the magnetic field, $\mu_{0}$ is the magnetic permeability (in free space $\left.\mu_{0}=4 \pi \times 10^{-7} \mathrm{H} / \mathrm{m}\right), M$ is the Magnetization.

The temperature, angular momentum, and magnetization relaxation equations are

$$
\begin{aligned}
& {\left[\rho C_{V, H}-\mu_{0} \boldsymbol{H} \cdot\left(\frac{\partial \boldsymbol{M}}{\partial T}\right)_{V, H}\right] \frac{D \boldsymbol{T}}{D t}+\mu_{0} T\left(\frac{\partial \boldsymbol{M}}{\partial T}\right)_{V, H} \cdot \frac{D \boldsymbol{H}}{D t}=K_{T} \nabla^{2} \mathrm{~T}} \\
& \rho \frac{D \boldsymbol{S}}{D t}=(\lambda+\eta) \nabla(\nabla \cdot \boldsymbol{\Omega})+\eta \nabla^{2} \boldsymbol{\Omega}+2 \xi(\nabla \times \mathbf{u}-2 \boldsymbol{\Omega})+\mu_{0}(\mathbf{M} \times \mathbf{H}) \\
& \frac{D \boldsymbol{M}}{D t}=\boldsymbol{\Omega} \times \boldsymbol{M}-\frac{1}{\tau}\left(\boldsymbol{M}-M_{0} \frac{\boldsymbol{H}}{H}\right)
\end{aligned}
$$

These are similar to the equations employed in polar fluid theories [6] but are here augmented by the inclusion of the magnetic field. Equation (5) is the simpler form of the magnetization relaxation equation proposed by Shliomis and Morozov [3]. Apart from some minor details, the formal structure of the magnetization equation [3,4] is nearly similar even though these have been derived differently. Here $\lambda, \eta$ are spin viscosities, $S=I \Omega$, where $I$ is the sum of moments of inertia of the sphere per unit volume and $\Omega$ is the average velocity of their ordered rotation, $C_{V, H}$ is the heat capacity at constant volume, $K_{T}$ is the thermal conductivity, $T$ is the temperature, $\boldsymbol{H}$ is the magnetic field, $\boldsymbol{M}$ is the magnetization, $\boldsymbol{M}_{0} \frac{\boldsymbol{H}}{H}=\boldsymbol{M}_{e q}$ is the equilibrium magnetization, $\boldsymbol{B}$ is the magnetic flux density and $\tau$ is the Brownian relaxation time.

Maxwell's equations in the magneto static limit are

$\nabla \cdot \mathbf{B}=0, \nabla \times \mathbf{H}=\mathbf{0}, \mathbf{B}=\mu_{0}(\mathbf{H}+\mathbf{M})$

We also assume Boussinesq approximation for the density variation and write $\rho=\rho_{0}\left[1-\alpha\left(T-T_{a}\right)\right]$

where $\alpha$ is the thermal expansion coefficient, $\rho_{0}$ is the mass density and $T_{a}$ is the average temperature. 
We assume that the equilibrium magnetization $M_{e q}$ is given by Langevin formula

$\mathbf{M}_{\mathrm{eq}}=\frac{\mathbf{H}}{\mathrm{H}} \mathrm{M}_{\mathrm{s}} \mathrm{L}\left(\alpha_{\mathrm{L}}\right)=\frac{\mathbf{H}}{\mathrm{H}} \mathrm{M}_{\mathrm{eq}}(\mathrm{H}, \mathrm{T}), \mathrm{L}\left(\alpha_{\mathrm{L}}\right)=\operatorname{coth}\left(\alpha_{\mathrm{L}}\right)-\frac{1}{\alpha_{\mathrm{L}}}, \alpha_{\mathrm{L}}=\frac{\mu_{0} \mathrm{mH}}{\mathrm{k}_{\mathrm{B}} \mathrm{T}}$,

Where $M_{S}$ the saturation magnetization, $\mathrm{m}$ is the magnetic moment of one particle and $k_{B}$ is the Boltzmann constant. In the limit of low magnetic field $\left(\alpha_{\mathrm{L}}<1\right)$, we define the initial susceptibility as

$\chi=\frac{\mu_{0} \mathrm{mM}_{\mathrm{s}}}{3 \mathrm{k}_{\mathrm{B}} \mathrm{T}_{\mathrm{a}}}=\frac{\mu_{0} \mathrm{~m}^{2} \mathrm{~N}}{3 \mathrm{k}_{\mathrm{B}} \mathrm{T}_{\mathrm{a}}}$

where $N$ is the number of magnetic dipole per unit volume and $\mathrm{k}_{\mathrm{B}}=1.38 \times 10^{-23} \mathrm{~J} / \mathrm{K}^{-1}$.

We consider two horizontal plates of infinite extent which contain the ferrofluid and which are separated by a vertical distance $d$ apart. The temperatures of the upper and lower plates are maintained at $T_{1}$ and $T_{0}$ respectively. A uniform magnetic field is applied normal to the plates. The boundary conditions are:

$\mathrm{u}=0, \Omega=0$ on the rigid wall $\mathrm{T}=\mathrm{T}_{0}$ at $\mathrm{z}=-\frac{\mathrm{d}}{2}, \mathrm{~T}=\mathrm{T}_{1}$ at $\mathrm{z}=\frac{\mathrm{d}}{2} ; \mathrm{T}_{\mathrm{a}}=\frac{1}{2}\left(\mathrm{~T}_{0}+\mathrm{T}_{1}\right)$

The magnetic boundary conditions are that the tangential component of the magnetic field and normal component of magnetic induction are continuous across the boundary.

In the quiescent state, following Finlayson [13], we express $\mathrm{M}_{\mathrm{eq}}=\mathrm{M}_{\mathrm{a}}+\chi\left(\mathrm{H}-\mathrm{H}_{\mathrm{a}}\right)-\mathrm{K}_{1}\left(\mathrm{~T}-\mathrm{T}_{\mathrm{a}}\right)$

where $\chi=\left(\frac{\partial M}{\partial H}\right)_{H_{a}, T_{a}}$ and $K_{1}=\left(\frac{\partial M}{\partial T}\right)_{H_{a}, T_{a}}$ are susceptibility and pyromagnetic coefficients, respectively.

The quiescent state solution of the basic equations is

$$
\boldsymbol{u}_{\boldsymbol{b}}=0, \Omega_{b}=0, \mathrm{~T}_{\mathrm{b}}=\mathrm{T}_{\mathrm{a}}-\beta \mathrm{z}, \beta=\frac{\mathrm{T}_{0}-\mathrm{T}_{1}}{\mathrm{~d}}, \mathbf{H}_{\mathrm{b}}=\left(\mathrm{H}_{\mathrm{a}}-\frac{\mathrm{K}_{1} \beta \mathrm{z}}{1+\chi}\right) \mathbf{k}, \mathbf{M}_{\mathrm{b}}=\left(\mathrm{M}_{\mathrm{a}}+\frac{\mathrm{K}_{1} \beta \mathrm{z}}{1+\chi}\right) \mathbf{k}
$$

To study linear stability of the above solution we now perturb the system as $\boldsymbol{u}=\boldsymbol{u}_{\boldsymbol{b}}+\boldsymbol{u}^{\prime}, \boldsymbol{\Omega}=\boldsymbol{\Omega}_{\boldsymbol{b}}+\boldsymbol{\Omega}^{\prime}, \mathrm{T}=\mathrm{T}_{\mathrm{b}}+\theta, \mathbf{H}=\mathbf{H}_{\mathrm{b}}+\mathbf{H}^{\prime}, \mathbf{M}=\mathbf{M}_{\mathrm{b}}+\mathbf{M}^{\prime}, \mathrm{P}=\mathrm{P}_{\mathrm{b}}+\mathrm{P}^{\prime}$

This gives the following set of linearized perturbation equations (dropping prime)

$$
\begin{aligned}
& \nabla \cdot \boldsymbol{u}=\mathbf{0}, \\
& \rho \frac{\partial \boldsymbol{u}}{\partial t} \pm \nabla P+(\xi+\mu) \nabla^{2} \mathbf{u}+\rho \alpha g \theta \mathbf{k}+\mu_{0}\left(\mathrm{M}_{\mathrm{a}}+\frac{\mathrm{K}_{1} \beta \mathrm{z}}{1+\chi}\right) \frac{\partial \nabla \phi}{\partial \mathrm{z}}-\mu_{0} \frac{\mathrm{K}_{1} \beta}{1+\chi} \mathrm{M}_{\mathrm{z}} \mathbf{k} \\
& \rho \frac{\partial \boldsymbol{S}}{\partial t}=(\lambda+\eta) \nabla(\nabla . \mathbf{\Omega})+\eta \nabla^{2} \mathbf{\Omega}+2 \xi(\nabla \times \mathbf{u}-2 \mathbf{\Omega}) \\
& +\mu_{0}\left(\mathrm{M}_{\mathrm{a}}+\frac{\mathrm{K}_{1} \beta \mathrm{z}}{1+\chi}\right)\left(-H_{y} \boldsymbol{i}+H_{x} \boldsymbol{j}\right)+\mu_{0}\left(\mathrm{H}_{\mathrm{a}}-\frac{\mathrm{K}_{1} \beta \mathrm{z}}{1+\chi}\right)\left(M_{y} \boldsymbol{i}-M_{x} \boldsymbol{j}\right)
\end{aligned}
$$




$$
\begin{aligned}
\frac{\partial M}{\partial t}+\frac{\mathrm{K}_{1} \beta \mathrm{z}}{1+\chi} \mathrm{w} \hat{\mathbf{k}} & =\left(\mathrm{M}_{\mathrm{a}}+\frac{\mathrm{K}_{1} \beta \mathrm{z}}{1+\chi}\right)\left(-\Omega_{y} i+\Omega_{x} \boldsymbol{j}\right) \\
- & \frac{1}{\tau}\left(M-\chi_{2} H_{x} \boldsymbol{i}-\chi_{2} H_{y} \boldsymbol{j}-\chi_{2} H_{z} \boldsymbol{k}+K_{1} \theta \boldsymbol{k}\right)
\end{aligned}
$$

$\rho_{0} \mathrm{C}_{\mathrm{V}, \mathrm{H}} \frac{\partial \theta}{\partial \mathrm{t}}+\left[\frac{\mu_{0} \chi \mathrm{H}_{\mathrm{a}} \beta}{\mathrm{T}_{\mathrm{a}}} \mathrm{z}-\mu_{0} \chi \mathrm{H}_{\mathrm{a}}\right] \frac{\partial^{2} \phi}{\partial \mathrm{t} \partial \mathrm{z}}=\mathrm{K}_{\mathrm{T}} \nabla^{2} \theta+\left[\frac{\mu_{0} \chi^{2} \mathrm{H}_{\mathrm{a}}^{2} \beta^{2}}{(1+\chi) \mathrm{T}_{\mathrm{a}}^{2}} \mathrm{z}+\beta \rho_{0} \mathrm{C}_{\mathrm{V}, \mathrm{H}}-\frac{\mu_{0} \chi^{2} \mathrm{H}_{\mathrm{a}}^{2} \beta}{(1+\chi) \mathrm{T}_{\mathrm{a}}}\right] \mathrm{w}$

$\nabla \cdot \mathbf{M}+\nabla^{2} \phi=0$

Where $\mathbf{M}=\left(\mathrm{M}_{\mathrm{x}}, \mathrm{M}_{\mathrm{y}}, \mathrm{M}_{\mathrm{z}}\right), \mathbf{H}=\left(\mathrm{H}_{\mathrm{x}}, \mathrm{H}_{\mathrm{y}}, \mathrm{H}_{\mathrm{z}}\right), \quad \Omega=\left(\Omega_{x}, \Omega_{y} \Omega_{z}\right)$ and $\chi_{2}=\frac{\mathbf{M}_{\mathrm{a}}}{\mathrm{H}_{\mathrm{a}}} \cdot \mathrm{We}$ have also used $\mathbf{H}=\nabla \phi$ since $\nabla \times \mathbf{H}=\mathbf{0}$.

On introducing the following non-dimensional quantities and non-dimensional parameters

$$
\begin{aligned}
& (\mathrm{x}, \mathrm{y}, \mathrm{z})=\mathrm{d}\left(\mathrm{x}^{*}, \mathrm{y}^{*}, \mathrm{z}^{*}\right), \mathrm{t}=\frac{\mathrm{d}^{2}}{\kappa} \mathrm{t}^{*}, \mathrm{P}=\frac{\mu \kappa}{\mathrm{d}^{2}} \mathrm{P}^{*}, u=\frac{\kappa}{d} u^{*}, \quad \Omega=\frac{\kappa}{\mathrm{d}^{2}} \Omega^{*}, \quad \theta=\mathrm{d} \beta \theta^{*}, \mathbf{M}=\mathrm{M}_{\mathrm{a}} \mathbf{M}^{*} \\
& , \quad \mathbf{H}=\mathrm{H}_{\mathrm{a}} \mathbf{H}^{*}, \quad \kappa=\frac{\mathrm{K}_{\mathrm{T}}}{\rho_{0} \mathrm{C}_{\mathrm{V}, \mathrm{H}}}, \quad \operatorname{Pr}=\frac{\nu}{\kappa}, \quad \mathrm{Ra}=\frac{\rho_{0} \mathrm{~g} \alpha \beta \mathrm{d}^{4}}{\mu \kappa}, \quad \tau=\frac{\mathrm{d}^{2}}{\kappa} \tau^{*}, \quad \xi=\mu \xi_{1}, \quad I=\mathrm{d}^{2} \mathrm{I}_{1}, \\
& \eta=\mu \mathrm{d}^{2} \eta_{1}, \\
& \lambda=\mu \mathrm{d}^{2} \lambda_{1}, \mathrm{M}_{1}=\frac{\mu_{0} \beta \chi^{2} \mathrm{H}_{\mathrm{a}}^{2}}{\rho_{0} \mathrm{~g}_{\mathrm{a}} \mathrm{T}_{\mathrm{a}}^{2}(1+\chi)}, \mathrm{M}_{2}=\frac{\mu_{0} \chi^{2} \mathrm{H}_{\mathrm{a}}^{2}}{\rho_{0} \mathrm{C}_{\mathrm{V}, \mathrm{H}}(1+\chi) \mathrm{T}_{\mathrm{a}}}, \mathrm{M}_{3}=\frac{\mu_{0} \chi \mathrm{H}_{\mathrm{a}}^{2}}{\rho_{0} \mathrm{~g} \alpha \mathrm{dT}_{\mathrm{a}}}, \\
& \operatorname{Ra}_{\mathrm{m}}=\operatorname{Ra}_{\mathrm{M}} \mathrm{M}_{1}=\frac{\mu_{0} \beta^{2} \chi^{2} \mathrm{H}_{\mathrm{a}}^{2} \mathrm{~d}^{4}}{\mu \kappa \mathrm{T}_{\mathrm{a}}^{2}(1+\chi)}, \mathrm{M}_{4}=\frac{(1+\chi) \mathrm{M}_{1}}{\chi \mathrm{M}_{3}}=\frac{\beta \mathrm{d}}{\mathrm{T}_{\mathrm{a}}},
\end{aligned}
$$

Where $\kappa$ is the fluid thermal diffusivity. The relevant equations, after dropping the asterisks, take the form

$$
\begin{aligned}
& \nabla \cdot \boldsymbol{u}=0 \\
& \frac{1}{\mathrm{P}_{\mathrm{r}}} \frac{\partial \mathrm{u}}{\partial \mathrm{t}}=-\nabla \mathrm{P}+\left(1+\xi_{1}\right) \nabla^{2} \mathrm{u}+\mathrm{R}_{\mathrm{a}} \theta \mathrm{k}+\mathrm{M}_{3} \mathrm{R}_{\mathrm{a}}\left[\left(\frac{\chi_{2}}{\chi \mathrm{M}_{4}}+\frac{1}{1+\chi} \mathrm{z}\right) \frac{\partial \nabla \phi}{\partial \mathrm{z}}-\frac{\chi_{2}}{1+\chi} \mathrm{M}_{\mathrm{z}} \mathrm{k}\right] \\
& +2 \xi_{1} \nabla \times \Omega \\
& \frac{1}{P_{r}} \frac{\partial \boldsymbol{S}}{\partial t}=\left(\lambda_{1}+\eta_{1}\right) \nabla(\nabla . \mathbf{\Omega})+\eta_{1} \nabla^{2} \boldsymbol{\Omega}+2 \xi_{1}(\nabla \times \boldsymbol{u}-2 \mathbf{\Omega}) \\
& +M_{3} R_{a}\left(\frac{\chi_{2}}{\chi M_{4}}+\frac{1}{1+\chi} z\right)\left(-\frac{\partial \phi}{\partial y} \boldsymbol{i}+\frac{\partial \phi}{\partial x} \boldsymbol{j}\right)+\chi_{2} M_{3} R_{a}\left(\frac{1}{\chi M_{4}}-\frac{1}{1+\chi} z\right)\left(M_{y} \boldsymbol{i}-M_{x} \boldsymbol{j}\right) \\
& \frac{\partial \boldsymbol{M}}{\partial t}+\frac{\chi M_{4}}{\chi_{2}(1+\chi)} w \boldsymbol{k}=\left(1+\frac{\chi M_{4} Z}{\chi_{2}(1+\chi)}\right)\left(\Omega_{y} i-\Omega_{x} \boldsymbol{j}\right) \\
& -\frac{1}{\tau}\left(\boldsymbol{M}-\frac{\partial \phi}{\partial x} \boldsymbol{i}-\frac{\partial \phi}{\partial y} \boldsymbol{j}-\frac{\chi}{\chi_{2}} \frac{\partial \phi}{\partial z} \boldsymbol{k}+\frac{\chi M_{4}}{\chi_{2}} \theta \boldsymbol{k}\right) \\
& \frac{\partial \theta}{\partial \mathrm{t}}+\frac{(1+\chi) \mathrm{M}_{2}}{\chi}\left(\mathrm{z}-\frac{1}{\mathrm{M}_{4}}\right) \frac{\partial^{2} \phi}{\partial \mathrm{t} \partial \mathrm{z}}=\nabla^{2} \theta+\left(\mathrm{M}_{2} \mathrm{M}_{4} \mathrm{z}+1-\mathrm{M}_{2}\right) \mathrm{w} \\
& \chi_{2} \nabla \cdot \mathbf{M}+\nabla^{2} \phi=0
\end{aligned}
$$


We further non-dimensionalize the magnetic potential $\phi$ and the magnetization vector $\mathrm{M}_{\mathrm{z}}$ as $\phi=\left(\frac{\chi}{1+\chi} \mathrm{M}_{4}\right) \phi^{\prime}, \mathrm{M}_{\mathrm{z}}=\left(\frac{\chi}{1+\chi} \frac{\mathrm{M}_{4}}{\chi_{2}}\right) \mathrm{M}_{\mathrm{z}}^{\prime}$, respectively.

On taking curl curl of equation (21) and curl of equation (22), the vertical component of resulting equations become, respectively, as

$$
\begin{array}{r}
\frac{1}{\operatorname{Pr}} \frac{\partial}{\partial \mathrm{t}} \nabla^{2} \mathrm{w}=\left(1+\xi_{1}\right) \nabla^{4} \mathrm{w}+\operatorname{Ra}_{1}^{2} \theta-\frac{\mathrm{Ra}_{\mathrm{m}}}{1+\chi} \frac{\partial}{\partial \mathrm{z}} \nabla_{1}^{2} \phi-\frac{\mathrm{Ra}_{\mathrm{m}}}{1+\chi} \nabla_{1}^{2} \mathrm{M}_{\mathrm{z}}+2 \xi_{1} \nabla^{2} \Gamma \\
\frac{\mathrm{I}_{1}}{\operatorname{Pr}} \frac{\partial \Gamma}{\partial \mathrm{t}}=-2 \xi_{1} \nabla_{1}^{2} \mathrm{w}-4 \xi_{1} \Gamma+\eta_{1} \nabla^{2} \Gamma+\operatorname{Ra}_{\mathrm{m}}\left(\frac{\chi_{2}}{\chi \mathrm{M}_{4}}+\frac{\mathrm{z}}{1+\chi}\right) \nabla_{1}^{2} \phi \\
+\operatorname{Ra}_{\mathrm{m}}\left(\frac{1}{\chi \mathrm{M}_{4}}-\frac{\mathrm{z}}{1+\chi}\right)\left(\nabla^{2} \phi+\frac{\partial \mathrm{M}_{\mathrm{z}}}{\partial \mathrm{z}}\right)
\end{array}
$$

where $\nabla_{1}^{2}=\left(\partial^{2} / \partial \mathrm{x}^{2}\right)+\left(\partial^{2} / \partial \mathrm{y}^{2}\right)$

Taking the divergence of magnetization equation (23) and using equation (25), we obtain

$$
\frac{\partial}{\partial \mathrm{t}} \nabla^{2} \phi=\frac{\partial \mathrm{w}}{\partial \mathrm{z}}-\chi_{2}\left(\frac{1+\chi}{\chi \mathrm{M}_{4}}+\frac{\mathrm{z}}{\chi_{2}}\right) \Gamma-\frac{1}{\tau}\left[\left(1+\chi_{2}\right) \nabla_{1}^{2} \phi+(1+\chi) \frac{\partial^{2} \phi}{\partial \mathrm{z}^{2}}-(1+\chi) \frac{\partial \theta}{\partial \mathrm{z}}\right]
$$

Taking vertical component of equation (23), we have

$$
\frac{\partial \mathrm{M}_{\mathrm{z}}}{\partial \mathrm{t}}=-\mathrm{w}-\frac{1}{\tau}\left[\mathrm{M}_{\mathrm{z}}-\chi \frac{\partial \phi}{\partial \mathrm{z}}+(1+\chi) \theta\right]
$$

Equations (24), (26)-(29) are five equations for five variables $W, \Gamma, \Phi, \mathrm{M}_{\mathrm{z}}$ and $\vartheta$. In these equations, $\mathrm{Pr}$ is the Prandtl number, $\xi_{1}$ is non-dimensionalized vortex viscosity, $R a$ is the viscous Rayleigh number, $\chi$ is the susceptibility, $\eta_{l}$ is the nondimensionalized spin viscosity, $\tau$ is the non-dimensionalized magnetic relaxation and $M_{4}=\beta d / T_{a}$. Thus, in comparison to the problem in quasistationary theory, we now have three more parameters: $\xi_{1}, \eta_{1}$ and $\tau$ which influence the stability problem. We note that if we set $\xi_{1}=0$ in (26) and $\tau=0$ in (28) and rearrange the terms we recover the equations studied by Finlayson [13]. There are, however, no counterparts to equation (27), the angular momentum equation, and the magnetization equation (29).

In order to match the domain of Chebyshev pseudospectral-QZ method, we reset the present domain from $\left[-\frac{1}{2}, \frac{1}{2}\right]$ to $[-1,1]$ with coordinate transformation from $z$ to $2 \mathrm{z}$ in equations (24), (26)-(29).

We now perform the standard normal mode analysis and look for the solution of variables $\mathrm{w}, \Gamma, \phi, \mathrm{M}_{\mathrm{z}}, \theta$ in the form

$$
\left[w, \Gamma, \phi, M_{z}, \theta\right]=\left[w(z), \Gamma(z), \phi(z), M_{z}(z), \theta(z)\right] \exp \left[\omega t+i\left(k_{x} x+k_{y} y\right)\right]
$$

where $\omega$ represents the scale frequency of the wave propagating in the disturbance wave direction. 
On substituting (30) into equation (24), (26)-(29) in the new domain, we obtain

$$
\begin{aligned}
& \frac{\omega}{P_{r}}\left(4 D^{2}-k^{2}\right) w(z)=\left(1+\xi_{1}\right)\left(16 D^{4}-8 k^{2} D^{2}+k^{4}\right) w(z)+2 \xi_{1}\left(4 D^{2}-k^{2}\right) \Gamma(z) \\
& -\mathrm{k}^{2} \operatorname{Ra} \theta(\mathrm{z})+\frac{\mathrm{Ra}_{\mathrm{m}}}{1+\chi} \mathrm{k}^{2} \mathrm{D} \phi(\mathrm{z})+\frac{\mathrm{Ra}_{\mathrm{m}}}{1+\chi} \mathrm{k}^{2} \mathrm{M}_{\mathrm{z}}(\mathrm{z}) \\
& \omega \frac{I_{1}}{P_{r}} \Gamma(z)=-2 \xi_{1}\left(4 D^{2}-k^{2}\right) w(z)+\eta_{1}\left(4 D^{2}-k^{2}\right) \Gamma(z) \\
& -k^{2} R_{a_{m}}\left(\frac{\chi_{2}}{\chi M_{4}}+\frac{1}{(1+\chi)} z\right) \phi(z) \\
& -4 \xi_{1} \Gamma(\mathrm{z})+\mathrm{Ra}_{\mathrm{m}}\left(\frac{1}{\chi \mathrm{M}_{4}}-\frac{1}{2(1+\chi)} \mathrm{z}\right)\left[\left(4 \mathrm{D}^{2}-\mathrm{k}^{2}\right) \phi(\mathrm{z})+2 \mathrm{DM}_{\mathrm{z}}(\mathrm{z})\right] \\
& \omega\left(4 D^{2}-k^{2}\right) \phi(z)=2 D w(z)-\chi_{2}\left(\frac{1+\chi}{\chi M_{4}}+\frac{z}{2 \chi_{2}}\right) \Gamma(z)+\frac{2(1+\chi)}{\tau} D \theta(z) \\
& -\frac{1}{\tau}\left[4(1+\chi) \mathrm{D}^{2}-\left(1+\chi_{2}\right) \mathrm{k}^{2}\right] \phi(\mathrm{z}) \\
& \omega M_{z}(z)=w(z)-\frac{1}{\tau} M_{z}(z)+\frac{2 \chi}{\tau} D \phi(z)-\frac{(1+\chi)}{\tau} \theta(z) \\
& \omega \theta(z)+\omega \frac{(1+\chi) M_{2}}{\chi}\left(z-\frac{2}{M_{2}}\right) D \phi(z)+\left(4 D^{2}-k^{2}\right) \theta(z)+\left(1-M_{2}+\frac{1}{2} M_{2} M_{4} z\right) w(z)
\end{aligned}
$$

where $k^{2}=k_{x}^{2}+k_{y}^{2}$,

boundary conditions

$\mathrm{w}=0, \Gamma=0, \theta=0$, at $\mathrm{z}= \pm 1 ; \mathrm{M}_{\mathrm{z}}+2 \mathrm{D} \phi-\mathrm{k} \phi=0$, at $\mathrm{z}=-1 ; \mathrm{M}_{\mathrm{z}}+2 \mathrm{D} \phi+\mathrm{k} \phi=0$, at $\mathrm{z}=+1$;

and

$\mathrm{Dw}=0$, at $\mathrm{z}= \pm 1$, on rigid-rigid surface

$\mathrm{Dw}=0$, at $\mathrm{z}=-1, \mathrm{D}^{2} \mathrm{w}=0$, at $\mathrm{z}=+1$, on rigid-free surface

$\mathrm{D}^{2} \mathrm{w}=0$, at $\mathrm{z}= \pm 1$, on free-free surface

\section{Numerical Results and Conclusion}

Several methods are available for solving the eigenvalue problem (31)-(35), together with the boundary conditions (36). In our study we solve this system of equation by using the Chebyshev pseudospectral method. Since most of the calculations here are based upon the Ester I base fluid, we have adopted values of some physical quantities $T_{a}=298 \mathrm{~K}, M_{s}=15900 \mathrm{~A} / \mathrm{m}, \rho_{0}=1150 \mathrm{~kg} / \mathrm{m}^{3}, \mu=0.014 \mathrm{~kg} /(\mathrm{m} . \mathrm{s}), K_{T}=0.31 \mathrm{~kg} . \mathrm{m} /\left(\mathrm{K} . \mathrm{s}^{3}\right)$, $\alpha=8.1 \times 10^{-4}, \chi=0.4, \chi_{2}=0.35, \quad \operatorname{Pr}=146.2, \kappa=10^{-7} \mathrm{~m}^{2} / \mathrm{sec}$. For the remaining parameters we have adopted the values discussed and used by Schumacher et al. [25], as $\mathrm{I}=7.5 \times 10^{-17} \mathrm{~m}^{2}$ and $\eta=2 \times 10^{-15} \mathrm{~kg} \cdot \mathrm{m} \cdot \mathrm{s}^{-1}$. 


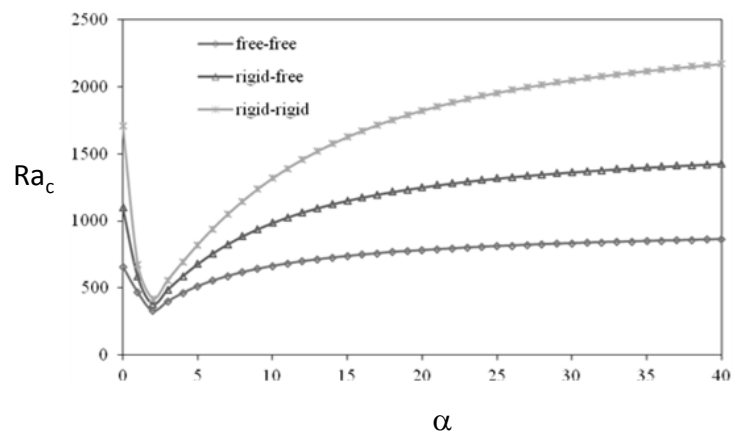

Fig. 1. Plot of $\mathrm{Ra}_{\mathrm{c}}$ with $\alpha_{\mathrm{L}}$ for Ester 1 base fluid and layer width $\mathrm{d}=1 \mathrm{~mm}$.

Following earlier writers Sunil and Mahajan [17], we have set $M_{2}=0$ in our calculations. In the following, $R a_{c}$ represents the critical Rayleigh number, $R a_{m, c}$ is the critical magnetic Rayleigh number and $R a_{m f, c}$ is the critical magnetic Rayleigh number in the gravity free.

We remark that we have carried out calculations for $R a_{c}$ and $R a_{m, c}$ for different values of $d$, for the Ester I base ferrofluid, but noticed that significant variation occurred only for $d=1 \mathrm{~mm}$. Our all subsequent calculation in this section are thus for $d=1 \mathrm{~mm}$. Moreover, the values considered for $\xi_{1}$ and $\tau$ are dimensionless values throughout.

In order to have some idea about the values of the critical parameters in different boundary conditions at the two plates, we have carried out calculations and have plotted them in Figs. 1-3. The three commonly used boundary condition are : free-free, rigid-free and rigid-rigid, and the fluid selected is Ester I base ferrofluid. The solution, in each case, has been obtained numerically. In general it is noted from the Figures that the pattern of values remains similar to be obtained in the case of a viscous fluid. The critical values are the lowest for the free-free boundary condition and the highest for the rigid-rigid case. Moreover, the critical values of $R a_{c}$ and $R a_{m, c}$ are field-dependent.

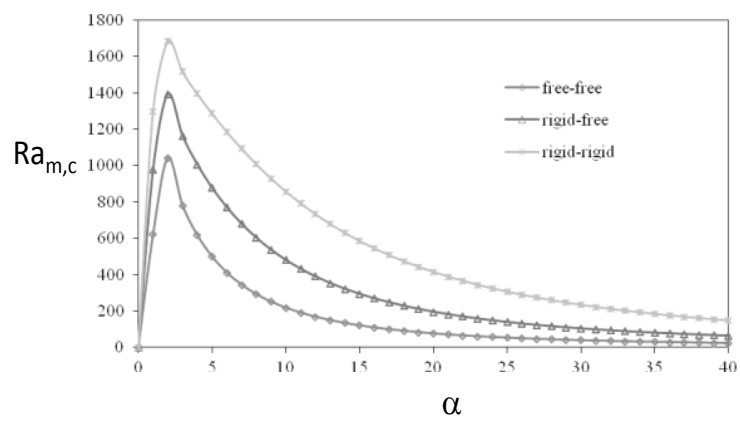

Fig. 2. Plot of $\mathrm{Ra}_{\mathrm{m}, \mathrm{c}}$ with $\alpha_{\mathrm{L}}$ for Ester 1 base fluid and layer width $\mathrm{D}=1 \mathrm{~mm}$. 
It is of some interest to determine the stability character in an environment when gravity effects can be neglected, i.e. when effects of magnetic field alone are present (e.g. international apace station). In the case when magnetic mechanism alone is considered to induce convection, the values of $R a_{m f, c}$, the critical magnetic Rayleigh number (in the microgravity environment), for three boundary conditions, in the case of I base ferrofluid are displayed in Fig. 3. Here again, at lower values of $\alpha_{L}$ the $R a_{m f, c}$ values steadily increase and then take nearly constant values. These $R a_{m f, c}$ values are almost identical, for all the three boundary conditions, at lower values of $\alpha_{L}$. However, as $\alpha_{L}$ is increased, it seems that the magnetization starts getting saturated and some structural changes begin. $R a_{m f, c}$ now takes on different values for the three boundary conditions and, as before, the convection starts at the lowest value of $R a_{m f, c}$ for freefree boundary condition. As compared to the pure viscous at $\alpha_{L}=10$, the values are 1598.9, 2085.1 and 2570.6, respectively, for the three boundary conditions. For higher values of $\alpha_{L}$, a slight change in $R a_{m f, c}$ only occurs in the case of rigid-rigid boundary condition.

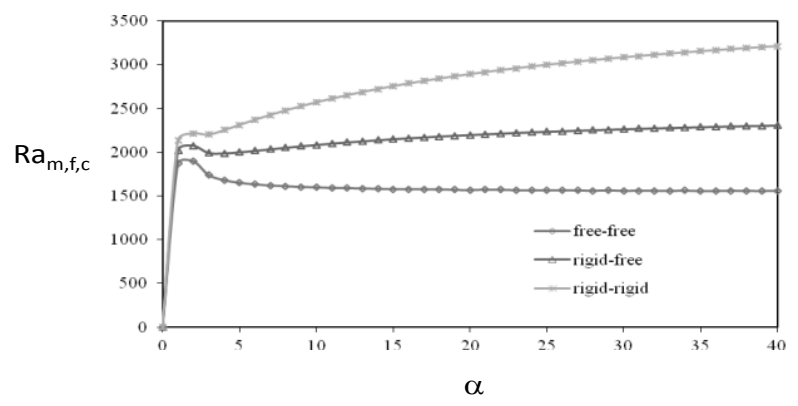

Fig. 3. Plot of critical $\mathrm{Ra}_{\mathrm{m}, \mathrm{f}, \mathrm{c}}$ (microgravity environment) with $\alpha_{\mathrm{L}}$ for different boundary conditions for Ester 1 base fluid and layer width $\mathrm{d}=1 \mathrm{~mm}$.

We note that corresponding values for a viscous fluid are: 657.5, 1101 and 1707.76, respectively, for the three boundary conditions. Thus it is clear that a ferrofluid in microgravity environment is more stable, not only as compared to the case when gravity present, but also to the pure viscous case. Finally, we also have noted that, as compared to the viscous fluid case, a higher temperature gradient is required for the threshold of convection in a microgravity environment.

\section{References}

1. R. E .Rosensweig, Ferrohydrodynamics (Dover Publications, Mineola, New York, 1997).

2. R. E. Rosensweig, R. Kaiser, and G. Miskolczy, J. Colloid Interface Sci. 29, 680 (1969). http://dx.doi.org/10.1016/0021-9797(69)90220-3

3. M. I. Shliomis and K .I. Morozov, Phys. Fluids 6(8), 2855 (1994). http://dx.doi.org/10.1063/1.868108 
4. B. U. Felderhof and H. J. Kroh, J. Chem. Phys. 110, 7403 (1999). http://dx.doi.org/10.1063/1.478642

5. J. S. Dahler and L. E. Scriven, Proc. Roy. Soc. A 275, 504 (1963). http://dx.doi.org/10.1098/rspa.1963.0183

6. D. W. Condiff and J. S. Dahler, Phys. Fluids 7, 842 (1964). http://dx.doi.org/10.1063/1.1711295

7. K. Shizawa and T. Tanahashi, J. Magn. Magn. Mater. 65, 181 (1987). http://dx.doi.org/10.1016/0304-8853(87)90027-8

8. S. R. deGroot and P. Mazur, Non-Equilibrium Thermodynamics (Dover, Mineola, New York, 1984).

9. R. E. Rosensweig, J. Chem, Phys. 121, 1228 (2004). http://dx.doi.org/10.1063/1.1755660

10. J. L. Neuringer and R. E. Rosensweig, Phys. Fluids 7, 1927 (1964). http://dx.doi.org/10.1063/1.1711103

11. P. J. Stile, F. Lin, and P. Blennerhassett, J. Colloid Interface. Sci. 151, 95 (1992). http://dx.doi.org/10.1016/0021-9797(92)90240-M

12. S. Chandrasekhar, Hydrodynamic and Hydromagnetic Stability (Dover, New York, 1981).

13. B. A. Finlayson, J. Fluid Mech. 40, 753 (1970). http://dx.doi.org/10.1017/S0022112070000423

14. K. Gotoh and M. Yamada, J. Phys. Soc. Japan 51, 3042 (1982). http://dx.doi.org/10.1143/JPSJ.51.3042

15. P. J. Stiles, and M. J. Kagan, J. Magn. Magn Mater. 85, 196 (1990). http://dx.doi.org/10.1016/0304-8853(90)90050-Z

16. P. Blennerhassett, F. Lin, and P. Stiles, Proc. Roy. Soc. London A 433, 165 (1991). http://dx.doi.org/10.1098/rspa.1991.0041

17. Sunil and A. Mahajan, Proc. Roy. Soc. A 464, 83 (2008). http://dx.doi.org/10.1098/rspa.2007.1906

18. M. D. Gupta and A. S. Gupta, Int. J. Eng. Sci. 17, 271 (1979). http://dx.doi.org/10.1016/0020-7225(79)90090-9

19. S. Venkatasubramanian and P. N. Kaloni, Int. J. Eng. Sci. 32, 237 (1994). http://dx.doi.org/10.1016/0020-7225(94)90004-3

20. L. M. Pérez, J. Bragard, D. Laroze, J. Martinez-Mardones, and H. Pleiner, J. Magn. Magn. Mat. 323, 691 (2011). http://dx.doi.org/10.1016/j.jmmm.2010.10.022

21. P. N. Kaloni and J. X. Lou, Phys. Rev. E 71, 066311 (2005). http://dx.doi.org/10.1103/PhysRevE.71.066311

22. P. Matura and M. Lücke, Phys. Rev. E 80, 026314 (2009). http://dx.doi.org/10.1103/PhysRevE.80.026314

23. L. Schwab, U. Hildebrandt, and K. Stierstadt, J. Magn. Magn. Mater. 39, 113 (1983). http://dx.doi.org/10.1016/0304-8853(83)90412-2

24. M. I. Shliomis, In Lecture Notes in Physics, Vol 584, ed. W. Kohler et al. (Springer, Berlin, 2002) 355.

25. A. Ghofrani, M. H. Dibaei, A. H. Sima, and M. B. Shafii, J. Expthermflusci. 49, 193 (2013).

26. S. Radha, S. Mohan, and C. Pai, J. Phys. B 448, 341 (2014). http://dx.doi.org/10.1016/j.physb.2014.04.050

27. H. A. Jasmine, J. Sci. Res. In press (2016). 